Mappemonde

Revue trimestrielle sur l'image géographique et les formes du territoire

$129 \mid 2020$

Varia

\title{
Atlas social de la métropole nantaise. Coordonné par François Madoré et Jean Rivière
}

Pierre Pistre

\section{OpenEdition}

Journals

Édition électronique

URL : https://journals.openedition.org/mappemonde/4491

DOI : 10.4000/mappemonde.4491

ISSN : 1769-7298

Éditeur

UMR ESPACE

\section{Référence électronique}

Pierre Pistre, «Atlas social de la métropole nantaise. Coordonné par François Madoré et Jean Rivière », Mappemonde [En ligne], 129 | 2020, mis en ligne le 10 novembre 2020, consulté le 18 novembre 2022. URL : http://journals.openedition.org/mappemonde/4491 ; DOI : https://doi.org/ 10.4000/mappemonde.4491

Ce document a été généré automatiquement le 18 novembre 2022.

\section{cc) (†)}

Creative Commons - Attribution - Pas d'Utilisation Commerciale - Partage dans les Mêmes Conditions 4.0 International - CC BY-NC-SA 4.0

https://creativecommons.org/licenses/by-nc-sa/4.0/ 


\title{
Atlas social de la métropole nantaise. Coordonné par François Madoré et Jean Rivière
}

\author{
Pierre Pistre
}

1 Lien vers le site $:$ https://asmn.univ-nantes.fr/

2 Après l'Atlas social d'Athènes recensé par Guy Burgel dans la rubrique Internet du numéro 126 (https://journals.openedition.org/mappemonde/1205), voilà une production en ligne plus que bienvenue sur une grande agglomération française, l'Atlas social de la métropole nantaise! Comme son "équivalent » grec, il s'agit d'un site qui publie une collection de notices - illustrées surtout par des planches cartographiques -, auxquelles on accède par le biais de différentes clés d'entrée (dernières publications, thèmes, mots clés...), et qui concilie particulièrement bien les enjeux de rigueur et de vulgarisation scientifique. Pour illustrer l'intérêt de ce projet récent d'édition électronique, cette recension présentera successivement ses objectifs et son organisation, puis mentionnera ses principales qualités et quelques limites ou pistes de développement.

\section{Un atlas en ligne pour « éclairer les réalités sociales qui traversent la métropole nantaise »}

Lancé à l'automne 2019, ce projet collaboratif en sciences sociales associe des géographes, des sociologues, des historiens, des aménageurs... sous la coordination de François Madoré et Jean Rivière, tous deux enseignants-chercheurs à l'Université de Nantes et au sein de l'UMR ESO (Espaces et Sociétés). Les objectifs sont clairement affichés dans le descriptif du projet: "éclairer les réalités sociales qui traversent la métropole nantaise », avec la volonté plus générale « d'interroger la dimension spatiale des inégalités sociales dans les mondes urbains» et «de comprendre les structures d'organisation de l'espace urbain et métropolitain ". Le cas nantais apparaît aussi très pertinent pour analyser les « effets des politiques d'attractivité » au cœur des principes 
contemporains de l'action publique métropolitaine, puisqu'il conserve une place sur le podium des métropoles françaises en croissance démographique depuis au moins le début des années 2000 (Vallès, 2019).

Pour satisfaire à ces objectifs, l'Atlas social de la métropole nantaise repose sur un principe de base, la publication de planches présentées sur la page d'accueil par date de mise en ligne (figure 1); environ 25 planches sont accessibles au moment de la publication de cette recension. Écrites par un ou plusieurs chercheurs - avec l'appui d'ingénieurs de l'Université de Nantes et du CNRS (Christophe Batardy, Simon Charrier, Stéphane Loret) -, elles présentent dans un format court (environ 5000 signes) et richement illustré ( 2 ou 3 cartes et graphiques par planche) une synthèse de résultats sur des thématiques variées. Au printemps 2020, Jean-Baptiste Bahers et ses coauteurs font par exemple un point sur les inégalités d'impacts écologiques (production de déchets, consommation d'énergies fossiles) dans les EPCI de la métropole, alors qu'Isabelle Garat analyse finement les inégalités de revenus dans l'espace métropolitain à partir des données du Fichier localisé social et fiscal (FiLoSoFi, INSEE). Les deux coordinateurs du projet sont aussi très impliqués dans la rédaction de planches sur leurs spécialités de recherche: François Madoré principalement sur la composition des ménages (personnes seules, familles monoparentales...) et les structures de logement (rues privées, résidences séniors...), Jean Rivière sur les résultats électoraux (municipales de 2014 et $2020 . .$.$) et les groupes socioprofessionnels. Citons également, à titre d'exemple,$ les caractéristiques de Nantes comme ville culturelle et touristique examinées dans deux planches par Basile Michel - dans la continuité de sa thèse sur les quartiers créatifs, dont celui des Olivettes (2017) -, ou la géographie des hommes et des femmes au quotidien analysée par Julie Vallée à partir du Mobiliscope, un outil Internet de géovisualisation déjà présenté dans le $\mathrm{n}^{0} 123$ de Mappemonde ( https:// journals.openedition.org/mappemonde/522). 


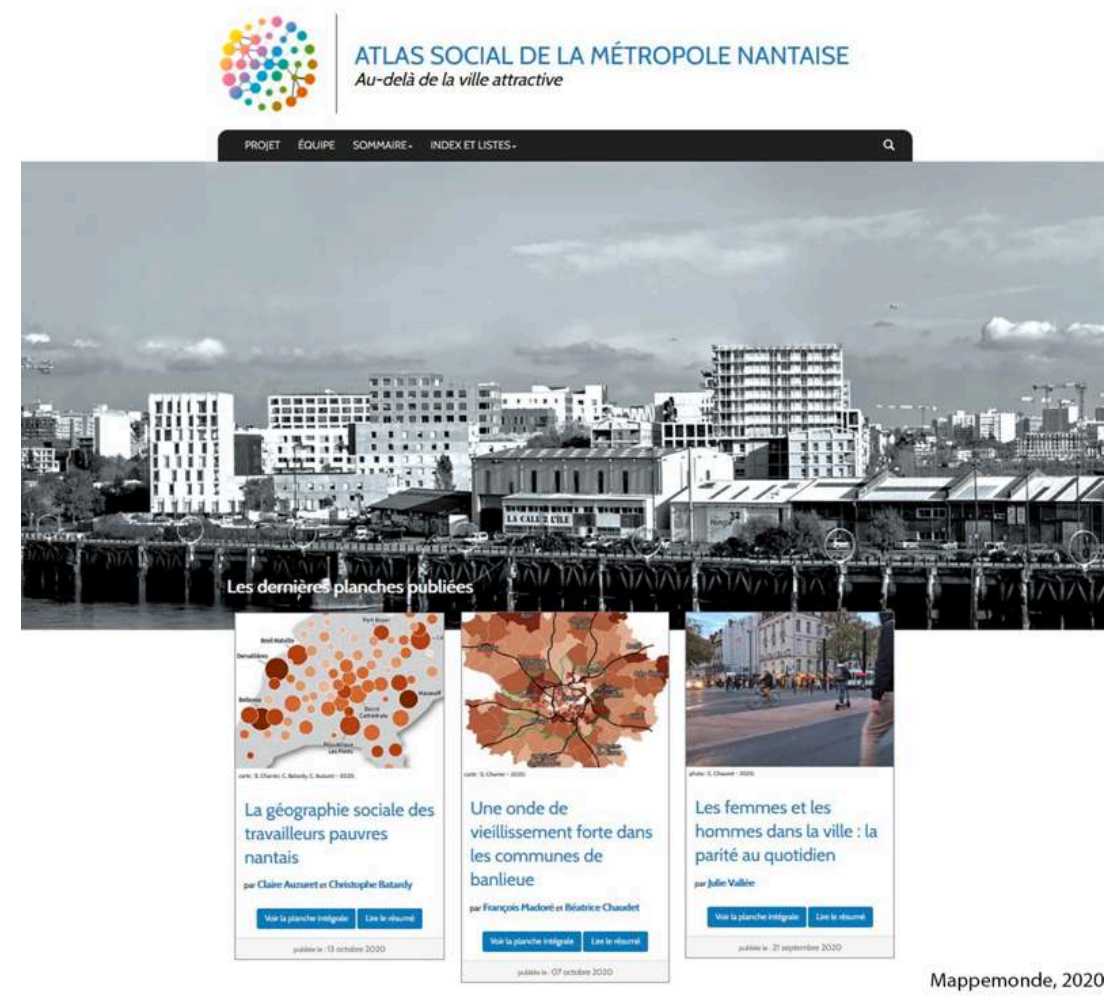

\section{Méthodes, technique, esthétique : différents choix pour une vulgarisation scientifique de qualité}

5 La variété des thèmes traités, associée à la qualité des contributeurs, est un des principaux intérêts de l'Atlas social de la métropole nantaise. À cela s'ajoute la rigueur méthodologique des analyses, un dispositif technique adapté aux objectifs et l'esthétique générale du site.

De nombreuses sources de données statistiques sont mobilisées (recensement de la population, résultats électoraux du ministère de l'Intérieur, données en opendata des services de Nantes Métropole...) pour objectiver les réalités sociales et spatiales de la métropole nantaise. Un souci particulier est donné à la précision spatiale des analyses : l'IRIS ou le bureau de vote sont notamment privilégiés pour les cartes thématiques infracommunales lorsque les données sont disponibles. D'autres planches, moins nombreuses, reposent sur des enquêtes qualitatives et sont illustrées par des cartes précises des lieux ou quartiers étudiés dans la ville (figure 2). Ainsi, par la variété des méthodes et des outils utilisés, cet atlas en ligne offre une image pluriscalaire de l'agglomération nantaise, de ses réalités (micro-)locales à sa géographie métropolitaine élargie au-delà du périmètre de Nantes Métropole (figure 3), voire en replaçant le cas d'étude dans l'espace français et international (cf. une planche de François Madoré sur le trafic aérien de l'aéroport de Nantes-Atlantique, figure 4). 
Figure 2. Exemples d'illustrations locales sur le marché de Talensac et la réserve foncière de la Beaujoire

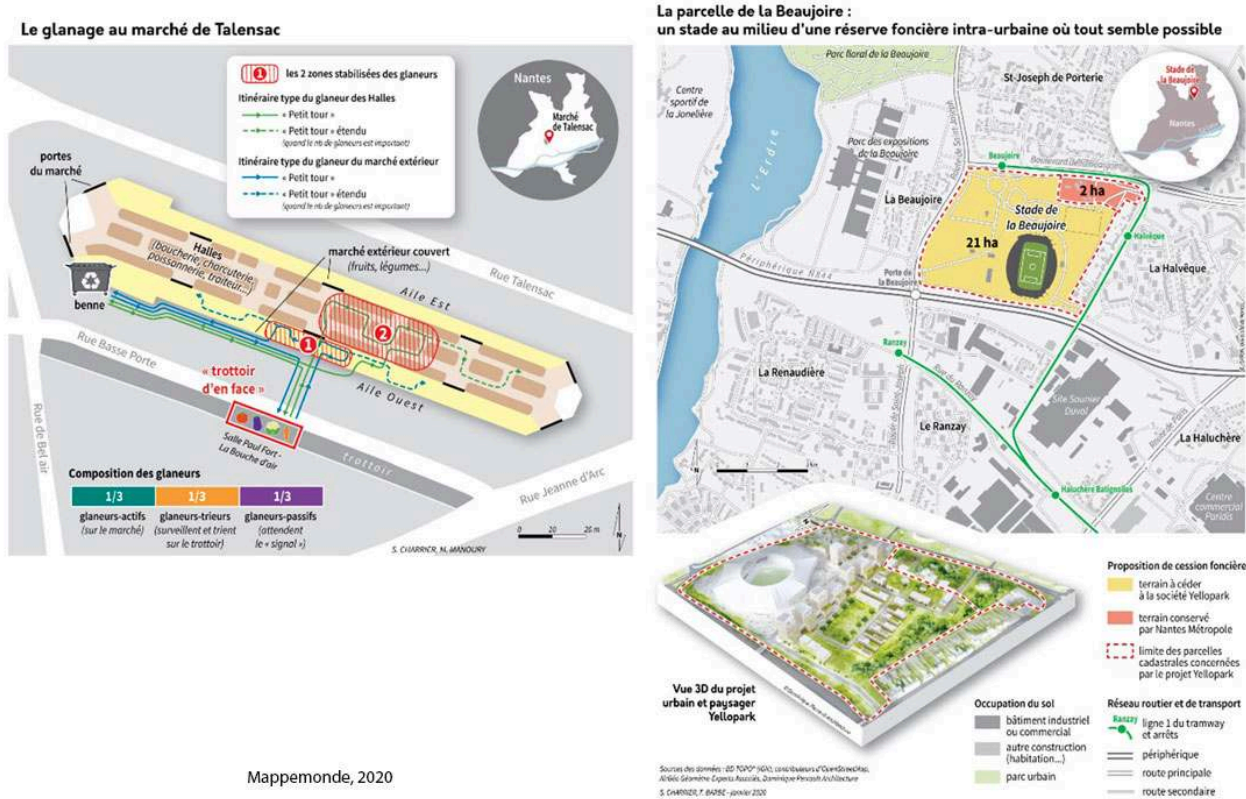

Talensac : Simon Charrier et Martin Manoury ; la Beaujoire : Simon Charrier et Frédéric Barbe

Figure 3. Exemple d'illustration associant plusieurs mailles et échelles de représentation sur les structures socioprofessionnelles dans l'Aire urbaine de Nantes

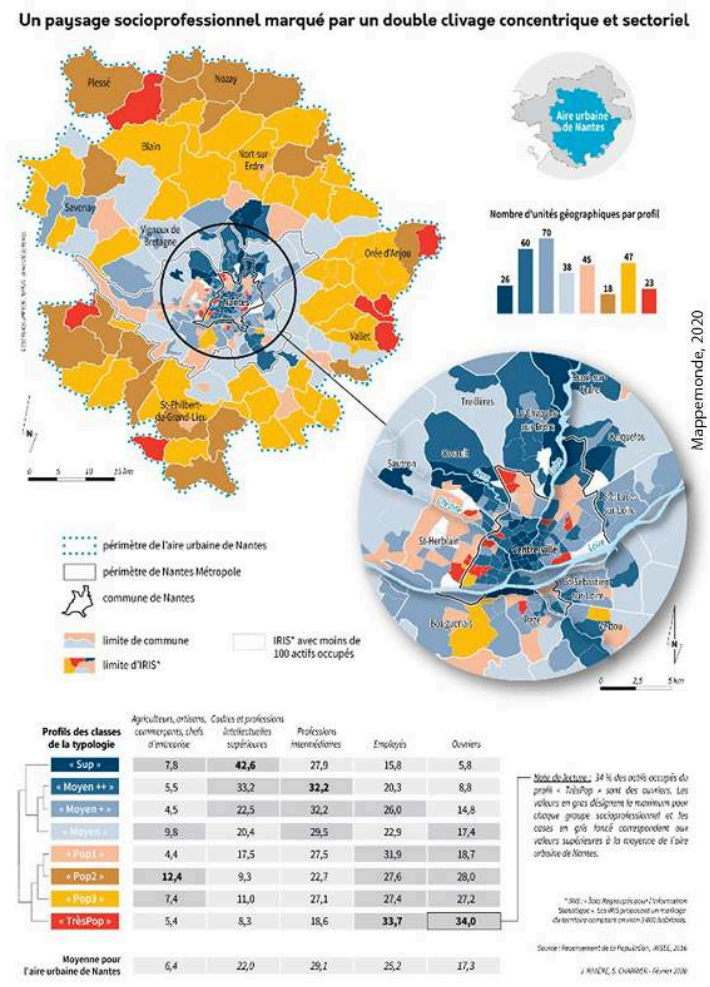

Jean Rivière et Simon Charrier 
Figure 4. Villes étrangères desservies par l'aéroport Nantes-Atlantique

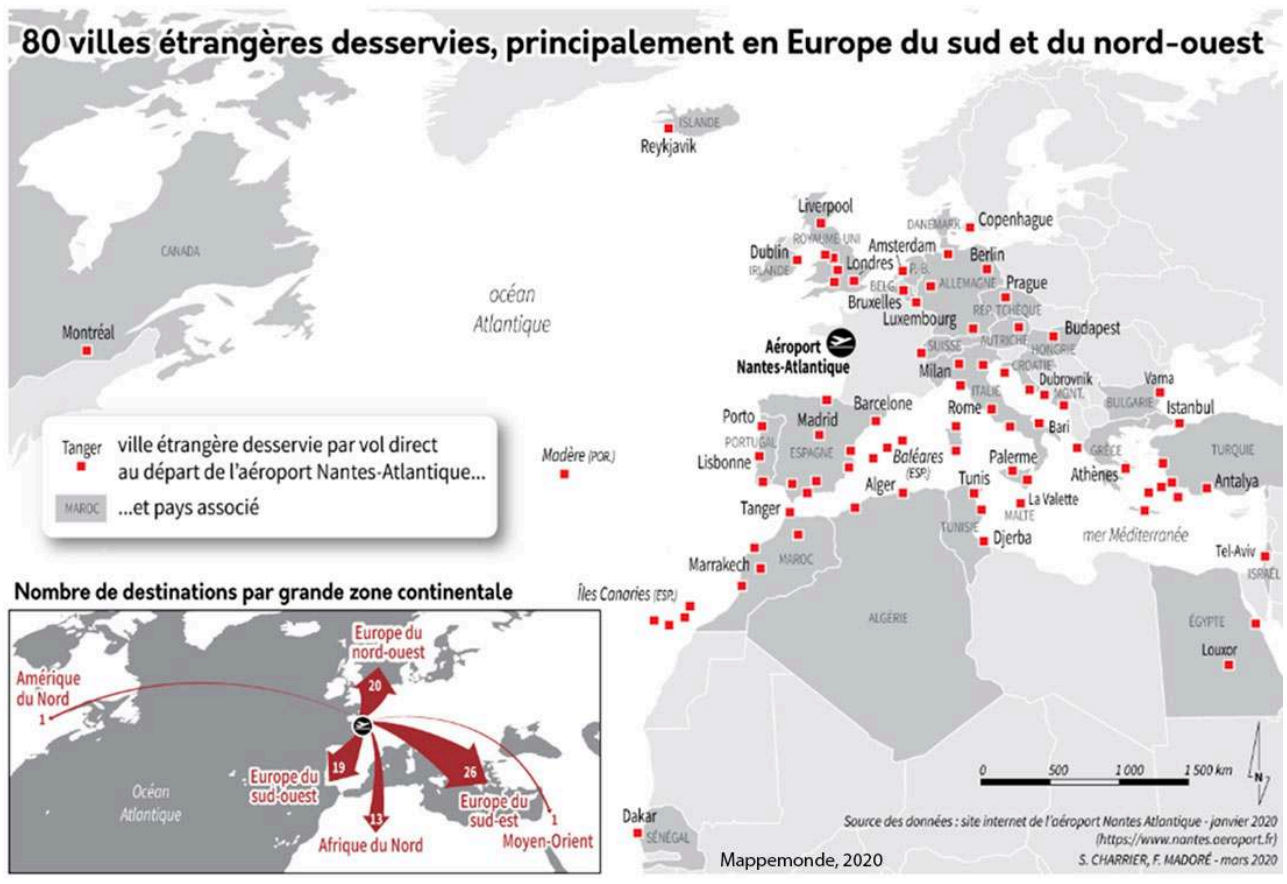

Simon Charrier et François Madoré

7 Une piste de développement pour prolonger les efforts conjoints de rigueur et de vulgarisation scientifique, le site pourrait renvoyer (encore) plus facilement des lecteurs extérieurs au monde de la recherche académique, mais très intéressés par telle ou telle planche, vers une publication scientifique du ou des auteurs; en l'état, des références sont parfois citées en bibliographie, mais pas systématiquement. Pourquoi ne pas imaginer une rubrique « pour aller plus loin» en fin de planche, qui renverrait vers une référence extérieure - en supplément des liens internes à l'atlas -, avec un minimum de présentation et idéalement sur des supports de publication en accès libre? Prenons pour exemple la synthèse éclairante de Boris Mericskay et Sylvain Grisot sur BlaBlaCar à Nantes (figure 5), un renvoi explicité vers l'article du premier auteur dans Cybergeo, sur le même sujet, serait assurément de nature à sensibiliser un plus large lectorat aux enjeux méthodologiques et thématiques de l'analyse des déplacements en France par covoiturage (Mericskay, 2019). 
Figure 5. Représentation cartographique d'une semaine de BlaBlaCar à Nantes

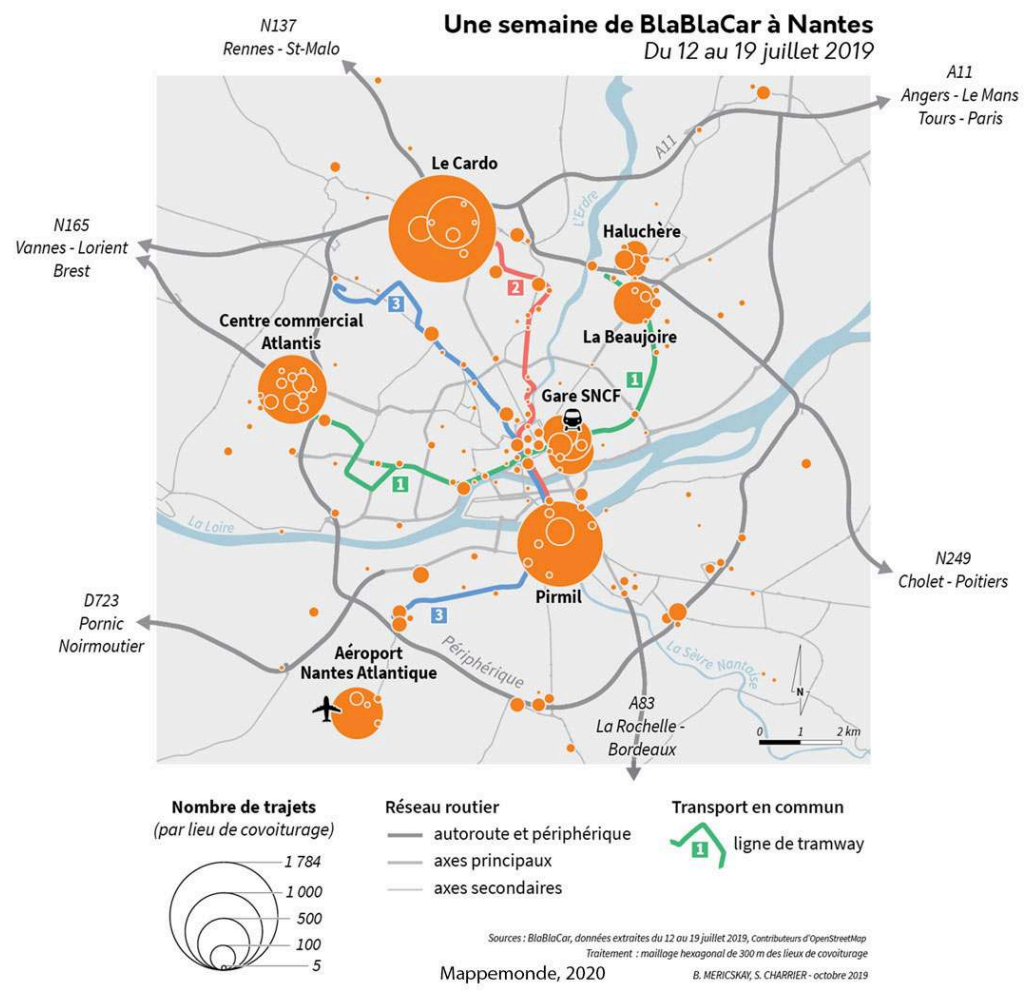

Boris Mericskay et Simon Charrier

8 Les concepteurs ont par ailleurs choisi Lodel - système de gestion de contenu (CMS) internet libre - pour créer et organiser l'Atlas social de la métropole nantaise. Tout à fait adapté pour la gestion des notices composées de textes et d'illustrations statiques, ce système assure une navigation aisée entre les différentes parties du site et les planches au cœur du fonctionnement de cet atlas en ligne (cf. sommaire, index géographique...); le «mur » de la page d'accueil permet aussi un accès facile à l'actualité de publication du site. Un léger regret technique, tout de même, concerne la quasi-absence de cartes interactives : l'atlas vise en premier lieu la production de synthèses finalisées, et le CMS choisi permet difficilement de gérer des données localisées (attributs, visualisation...); mais si l'on considère que l'introduction de contenus dynamiques est une des plusvalues principales d'un atlas en ligne - y compris pour "naviguer» sur des cartes thématiques et accéder facilement à des informations utiles (nom des unités géographiques, valeurs statistiques représentées...) -, le caractère statique des documents peut parfois frustrer le lecteur, plus ou moins averti. La première planche de l'atlas publiée en novembre 2019 sur les rues privées incluait d'ailleurs un module d'exploration à l'échelle de la métropole nantaise (figure 6). 
Figure 6. Module d'exploration des voies privées dans la métropole nantaise

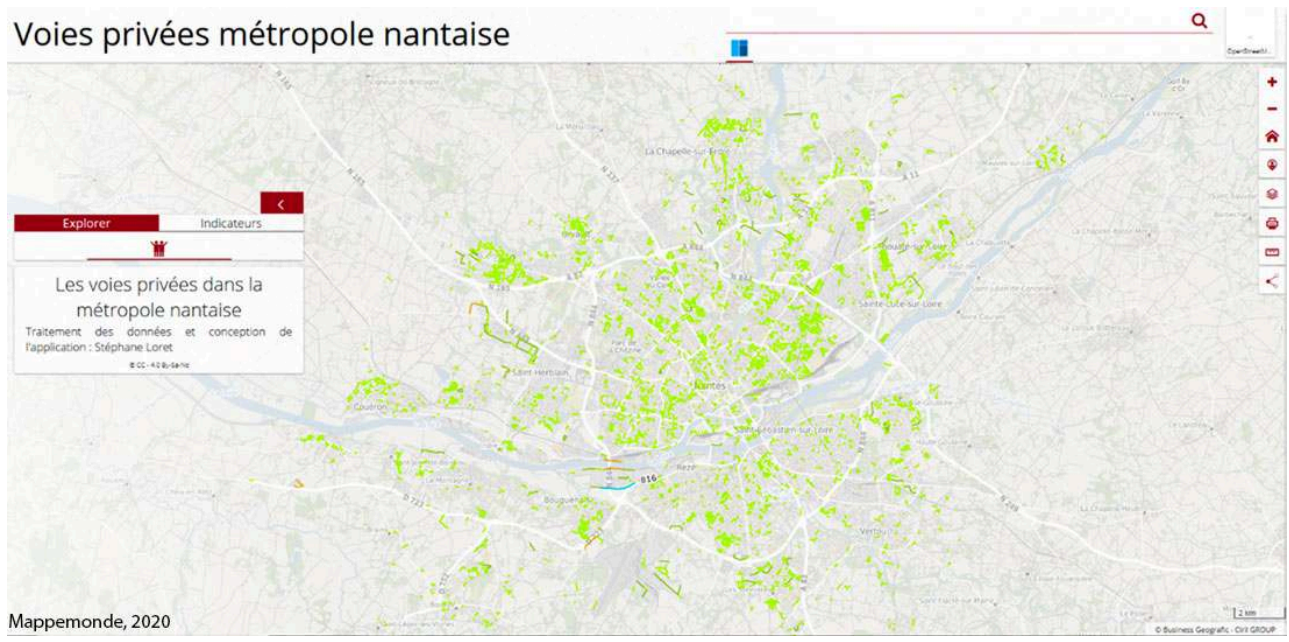

Sur un plan plus esthétique, il faut également souligner l'attrait général du site et sa clarté. Les concepteurs ont fait l'effort d'une charte graphique homogène, pour le texte (police des titres, organisation des paragraphes...) ainsi que les figures, notamment les cartes (cf. échelle, nord...), avec tout de même une liberté d'application qui permet de personnaliser les réalisations. Malgré de rares représentations non harmonisées, l'Atlas social de la métropole nantaise bénéficie aussi d'une excellente qualité des illustrations, en premier lieu cartographiques, mais aussi photographiques ou même sonores dans une planche de Christophe Batardy sur les élections municipales de 1977 (cf. entretien avec Alain Chénard, ancien maire de Nantes). Une interrogation esthétique néanmoins : le logo de l'atlas est élégant, "l'arc en ciel» attire l'œil, mais quel sens porte-t-il (figure 1) ? Fait-il référence à la structuration sociodémographique de la métropole ou à sa subdivision communale ou en quartiers?

En définitive, le bilan de ce projet collaboratif d'édition électronique est plus que positif. Par ses qualités scientifiques, techniques, esthétiques, il peut notamment intéresser un grand nombre de publics: aussi bien des acteurs territoriaux que des citoyens comme outil d'information et de réflexion sur le fonctionnement de la métropole nantaise, des étudiants et des élèves du secondaire comme outil pédagogique, des curieux et voyageurs $\mathrm{du}$ web comme outil de pérégrinations géographiques à distance! Ainsi, comment ne pas souhaiter voir se développer des atlas en ligne équivalents sur d'autres métropoles françaises, associant les connaissances de chercheurs en géographie et sciences sociales et l'appui indispensable d'ingénieurs en cartographie, géomatique, informatique? En attendant, ne vous privez pas de découvrir, d'utiliser voire de participer - les contributions spontanées sont en effet possibles en prenant contact avec l'équipe éditoriale - à l'Atlas social de la métropole nantaise, dans sa version en ligne ou prochainement en version papier (Madoré et al., 2021). 


\section{BIBLIOGRAPHIE}

BURGEL G. (2019). « Atlas Social d'Athènes ». Mappemonde, nº 126. En ligne : https:// journals.openedition.org/mappemonde/1205

LECOMTE C., VALLÉE J., LE ROUX G., COMMENGES H. (2018). « Le Mobiliscope, un outil de géovisualisation des rythmes quotidiens des métropoles ». Mappemonde, $\mathrm{n}^{\circ} 123$. En ligne : https://

journals.openedition.org/mappemonde/522

MADORÉ F., RIVIÈRE J., BATARDY C., CHARRIER S., LORET S., dir. (2021). Atlas social de la métropole nantaise. $A u$-delà de la ville attractive. Presses universitaires de Rennes (à paraître).

MERICSKAY B. (2019). « Potentiels et limites des traces (géo)numériques dans l'analyse des mobilités : l'exemple des données de la plateforme de covoiturage BlaBlaCar ». Cybergeo. En ligne : https://journals.openedition.org/cybergeo/31990

MICHEL B. (2017). Les quartiers créatifs : une dynamique de club. Analyse croisée des quartiers des Olivettes (Nantes), du Panier (Marseille) et Berriat (Grenoble). Thèse de doctorat de géographie à l'Université d'Angers, 478 p. En ligne : https://tel.archives-ouvertes.fr/tel-01720500/file/ these_MICHEL_STAR.pdf

VALLÈs V. (2019). « Démographie des EPCI : la croissance se concentre dans et au plus près des métropoles ». Insee Première, $\mathrm{n}^{\circ}$ 1729, 4 p. En ligne : https://www.insee.fr/fr/statistiques/3694585

\section{AUTEUR}

\section{PIERRE PISTRE}

Université de Paris/UMR Géographie-cités 